\section{Can Our Choice of Contrast Media Impact Renal and Cardiovascular Outcomes?}

\author{
Anand Prasad, ${ }^{1}{ }^{*} \mathrm{MD}, \mathrm{FACC}, \mathrm{FSCAl}, \mathrm{RPVI}$, and \\ Hitinder S. Gurm, ${ }^{2}$ MBBS \\ ${ }^{1}$ University of Texas Health Science Center at San \\ Antonio, San Antonio, Texas \\ ${ }^{2}$ University of Michigan Health System, Ann Arbor, \\ Michigan
}

\section{Key Points}

- Contrast media vary in their ionic status, viscosity, osmolarity - and these factors all play into clinical effects.

- Iso-osmolar and low-osmolar contrast media dominate clinical use today. The differences between these two agents in terms of kidney injury remain uncertain.

- Ionic agents have theoretical properties which may help prevent thrombus formation; however this phenomenon has not translated to differences in clinical outcomes.

The choice of specific contrast media (CM) for invasive angiography remains controversial. Furthermore, despite the ubiquitous presence of $\mathrm{CM}$ in the catheterization laboratory, the clinical relevance of the underlying chemical properties of individual agents remains confusing at best. Fundamentally, the goal of angiography is to deliver adequate iodine molecules through a vessel to opacify the lumen. While seemingly straightforward, this goal can be accomplished in a variety of ways by altering the viscosity, osmolality, and concentration of iodine-based CM. In practice, we summarize the chemical nature of $\mathrm{CM}$ as ionic versus non-ionic and iso, low, or hyperosmolar.

The basic structure of modern $\mathrm{CM}$ relies on the six carbon benzoic acid ring, able to deliver three iodine atoms per molecule. Ionic agents can be a monomers or dimers with the size of the molecule relating to its viscosity. Ionic agents have both an anion (negatively charged) and a cation (positively charged) ions. The anion is either an iodamide, iothalamate, metrizoate, or diatrizoate ion, while the cation is either a sodium or meglumine ion. Ionic agents also contain carboxylate group (COO-) which helps improve hydrophilicity. The non-ionic agents have increased hydroxyl groups $(\mathrm{OH})$ to improve their solubility in water. The osmolarity of $\mathrm{CM}$ also has important clinical and chemical implications. Ultimately, the number of iodine atoms per particles in solution (CM ratio) helps describe the number of molecules that must be delivered to get the same relative effect of X-ray absorption. Examples include, high osmolar ionic monomers (CM ratio 1.5), versus non-ionic dimers (CM ratio 6). The earliest agents in clinical use were ionic, high osmolar contrast agents with non-ionic agents arriving in clinical use in the 1960s. The high osmolar agents were associated with significantly higher rates of adverse reactions compared to low osmolar agents and are now rarely used in the context of coronary angiography. At this point in time, iso-osmolar or low osmolar agents dominate use. The relative benefit of iso-osmolar agents over low osmolar media remain controversial-though some data would support lower rates of acute kidney injury (AKI),with iso-osmolar agents compared with some but not all low osmolar agents and less pain when used in the peripheral circulation.

Whether ionic agents offer any benefit in terms of renal toxicity or broader cardiovascular impact over non-ionic agents remains unclear. Ionic agents have some attractive theoretical properties. In ex vivo studies, ionic agents have been shown to have antiplatelet and anti-thrombotic activity. However, in-vivo studies have failed to uniformly demonstrate benefits on major adverse cardiac events [1]. The ICON trial, published in 2009, examined the comparative impact of ionic low-osmolar (ioxaglate) versus non-ionic iso-osmolar (iodixanol) on changes in serum creatinine following

Conflict of interest: Nothing to report.

*Correspondence to: Anand Prasad, MD, FACC, FSCAI, University of Texas Health Science Center San Antonio, 7703 Floyd Curl Drive, San Antonio, TX 78229. E-mail: prasada@uthscsa.edu

Received 1 February 2016; Revision accepted 7 February 2016

DOI: $10.1002 / \mathrm{ccd} .26483$

Published online 14 March 2016 in Wiley Online Library

(wileyonlinelibrary.com) 
coronary angiography and found no differences in contrast-induced AKI [2]. The study was a relatively small randomized controlled trial and underpowered for AKI. The present paper in Catheterization and Cardiovascular Interventions by Giustino et al. examines the 30-day and one year results of the ICON trial with respect to renal failure and mortality [3]. There were no differences in renal outcomes and a numerically (but not statistically significant) higher rate of death at one year in the iodixanol group versus the group ioxaglate $(9.1 \%$ vs. $2.7 \%, P=0.07)$. So what do we make of these results? It is hard to make any definitive conclusions, the numerically higher rates in the iodixanol group may be a trend-leading to hypothesis generation or more likely a play of chance due to a small underpowered study. If we are to work under the construct that ionic CM may have beneficial thrombotic properties then unfortunately the causes of death in the trial do not help shed light on this potential benefit as the etiologies of cardiovascular death in both groups were highly variable. Ultimately what the ICON trial really teaches us is the difficulty in designing trials to evaluate strategies to reduce AKI given the confounding impact of $\mathrm{CM}$ properties, hydration strategies, patient co-morbidities, and role of baseline volume status. For now, it is still hard to make the argument that use of a specific modern CM has overwhelming benefit over another.

\section{REFERENCES}

1. Bertrand ME, Esplugas E, Piessens J, Rasch W. Influence of a nonionic, iso-osmolar contrast medium (iodixanol) versus an ionic, low-osmolar contrast medium (ioxaglate) on major adverse cardiac events in patients undergoing percutaneous transluminal coronary angioplasty: A multicenter, randomized, double-blind study. Visipaque in Percutaneous Transluminal Coronary Angioplasty [VIP] Trial Investigators. Circulation 2000;101:131-136.

2. Mehran R, Nikolsky E, Kirtane AJ, Caixeta A, Wong SC, Teirstein PS, Downey WE, Batchelor WB, Casterella PJ, Kim $\mathrm{YH}$, et al. Ionic low-osmolar versus nonionic iso-osmolar contrast media to obviate worsening nephropathy after angioplasty in chronic renal failure patients: The ICON (Ionic versus non-ionic Contrast to Obviate worsening Nephropathy after angioplasty in chronic renal failure patients) study. JACC Cardiovasc Interv 2009;2:415-421.

3. Giustino G, Baber U, Mastoris I, Vlachojannis GJ, Yu J, Teirstein PS, Downey WE, Batchelor WB, Casterella PJ, Nikolsky E, et al. One-year results of the ICON (ionic versus non-ionic contrast to obviate worsening nephropathy after angioplasty in chronic renal failure patients) Study. Catheter Cardiovasc Interv 2016;87:703-709. 